Ann. Génét. Sél. anim., I979, 11 (I), 53-77.

\title{
Analyse des variations génétiques entre trois races de lapins de la taille de portée et de ses composantes biologiques en saillie post-partum
}

\author{
Françoise HULOT et G. MATHERON \\ Station d'Amélioration Génétique des Animaux \\ Centre de Recherches de Toulouse, I.N.R.A., \\ B.P. I2, 31320 Castanet-Tolosan
}

\section{Résumé}

Une laparotomie de 273 lapines primipares de 9 génotypes, saillies post partum par des mâles Fauves de Bourgogne, nous a permis de mesurer le nombre de corps jaunes, de sites d'implantation et d'embryons à $\mathrm{I} 6$ jours de gestation. Les 9 types génétiques sont constitués de 3 races pures (Californienne, Néo-Zélandaise Blanche, Petite Russe) et des 6 croisements réciproques obtenus par la combinaison deux à deux de ces 3 races. Un modèle de décomposition des effets génétiques directs, maternels et grand maternels sur la taille de portée et ses composantes (nombre d'ovules et viabilité embryonnaire) est présenté. La comparaison des effets génétiques en cause pour les composantes du taux de gestation et de la taille de portée montre la supériorité de la race Californienne pour les effets génétiques directs sur le nombre d'ovules, l'aptitude de la race Petite Russe pour les effets génétiques directs sur la gestation et la viabilité embryonnaire, et l'importance de l'effet favorable de l'ascendance maternelle Néo-Zélandaise d'une femelle pour la viabilité de ses cufs jusqu'à I 6 jours. Des effets d'hétérosis directs de l'ordre de ro p. I00 sont mis en évidence pour le nombre d'ovules. Des effets d'hétérosis maternels de ro à 20 p. 100 sont rapportés pour la seule viabilité pré-implantatoire. Un essai de discussion des effets génétiques estimés par analyse de variance est abordé en relation avec les mécanismes biologiques en cause.

Les performances de reproduction chez la lapine dépendent du nombre et de la taille des portées effectuées au cours de sa carrière de reproductrice. D'une part l'existence dans cette espèce d'un œstrus post partum très marqué chez une femelle allaitante suggère une intensification du rythme des accouplements pour accroître de façon sensible la productivité numérique (PRUD'HON et al., I969). D'autre part, la taille de portée est un caractère biologique trop global pour que sa sélection conduise à un rapide progrès (héritabilité généralement faible) ou que la prédiction de types de croisements soit à coup sûr judicieuse. L'analyse de la variabilité génétique de ses composantes (ovulation, implantation, viabilité fotale), sous la dépendance conjointe de groupes de gènes différents de la mère et des lapereaux,

Cette Recherche a été effectuée grâce au concours d'une action thématique programmée définie par la commission spécialisée lapin de l'I.N.R.A. (ATP, $n^{\circ} 65525$ ). 
paraît indispensable pour assurer une réelle maîtrise de l'amélioration de ce caractère complexe.

Cette étude a donc pour but essentiel la connaissance des effets génétiques en cause chez 9 génotypes de femelles primipares allaitantes saillies par des mâles d'une souche unique et différente, en période post partum. Ce mode de conduite permet d'effectuer 1'analyse dans des conditions extrêmes d'expression de la productivité numérique. Une étude globale de la fécondité de ces mêmes femelles en première mise bas complètera les résultats analytiques obtenus au cours de leur deuxième portée.

\section{Matériel et méthodes}

\section{I. - Les types génétiques utilisés}

Un plan factoriel de croisement entre 3 races, la Californienne, la Néo-Zélandaise et la Petite Russe, a été mis en place pour fournir les 9 génotypes expérimentaux. Ces animaux issus de troupeaux fermés actuellement sélectionnés à la Station d'Amélioration Génétique des Animaux, correspondent, pour les deux premières

TABLEAU I

Répartition des effectifs de femelles dans les 9 génotypes mis en comparaison issus des souches parentales pures: Californienne (CA) Néo-Zélandaise Blanche (NZ) Petite Russe (PR)

\begin{tabular}{|c|c|c|c|c|}
\hline${ }_{\text {Race mère }}$ Race père & CA & $\mathrm{NZ}$ & PR & Total \\
\hline $\mathrm{CA} \cdot \cdot \cdot$ & $\begin{array}{ll}23 & (1) \\
20 & (2) \\
\text { I } 5 & (3) \\
\text { I5 } & (4)\end{array}$ & $\begin{array}{l}27 \\
23 \\
22 \\
21\end{array}$ & $\begin{array}{l}24 \\
17 \\
17 \\
16\end{array}$ & $\begin{array}{l}74 \\
60 \\
54 \\
5^{2}\end{array}$ \\
\hline $\mathrm{NZ}$ & $\begin{array}{l}37 \\
28 \\
28 \\
27\end{array}$ & $\begin{array}{l}34 \\
21 \\
20 \\
20\end{array}$ & $\begin{array}{l}39 \\
36 \\
34 \\
34\end{array}$ & $\begin{array}{r}\text { I 10 } \\
85 \\
82 \\
81\end{array}$ \\
\hline PR & $\begin{array}{l}36 \\
33 \\
32 \\
31\end{array}$ & $\begin{array}{l}40 \\
36 \\
30 \\
29\end{array}$ & $\begin{array}{l}13 \\
13 \\
12 \\
12\end{array}$ & $\begin{array}{l}89 \\
82 \\
74 \\
72\end{array}$ \\
\hline Total & $\begin{array}{l}96 \\
81 \\
75 \\
73\end{array}$ & $\begin{array}{r}\text { IOI } \\
80 \\
72 \\
72\end{array}$ & $\begin{array}{l}76 \\
66 \\
63 \\
62\end{array}$ & $\begin{array}{l}273 \\
227 \\
210 \\
205\end{array}$ \\
\hline
\end{tabular}

Dans chaque case du tableau figurent :

(I) Nombre de femelles réalisant une mise bas $I$.

(2) Nombre de femelles ovulant à la saillie Post Partum.

(3) Nombre de femelles ayant des sites.

(4) Nombre de femelles ayant des embryons. 
races, à des reproducteurs de format moyen, utilisés pour la production de femelles croisées par les éleveurs, et pour la troisième à des animaux de petit format remarquables pour le taux de gestation qu'ils transmettent à leur descendance femelle. Soixante-quinze femelles accouplées à 24 mâles ont fourni au cours de leurs 5 premières portées, de mai I 974 à janvier 1975, les 273 femelles correspondant au schéma génétique défini ( 3 races pures et les 6 génotypes croisés correspondants dont les effectifs sont indiqués dans le tabl. I). Par ailleurs, un lot de I 9 mâles Fauves de Bourgogne, issu d'un troupeau extérieur, est mis en place pour réaliser toutes les saillies. Le but recherché est en effet de mettre en évidence les différences éventuelles entre génotypes maternels en les accouplant à une souche unique paternelle servant de "testeur commun".

\section{2. - Protocole expérimental}

Les accouplements de femelles nullipares, âgées de 4 mois $I / 2$ environ, ont été effectués entre le I5 novembre I974 et le 19 juin 1975 en vue d'obtenir une première portée. L'âge moyen à la sai!lie positive, de I47,4 \pm I,4 jours, n'est pas significativement différent pour chaque génotype. Les accouplements post partum ont été réalisés entre le 17 décembre 1974 et le 23 juillet I975. Pour chaque femelle, le choix du mâle pour les deux saillies a été effectué de façon aléatoire. L'obtention des animaux en âge de reproduire a été planifiée afin que chaque génotype soit également représenté tout au long des 2 périodes de 7 mois nécessaires à la réalisation complète de l'expérience.

Compte tenu des nombreux facteurs qui, en saillies post partum tout particulièrement, paraissent influer sur les performances de reproduction, un protocole rigoureux a été élaboré pour standardiser au maximum les conditions de milieu et l'état physiologique des animaux.

Les femelles sont élevées en cage individuelle dans un bâtiment clos, maintenu à une température d'environ $\mathrm{I} 8^{\circ} \mathrm{C}$. La luminosité naturelle est en période hivernale complétée par un système artificiel réalisant un éclairement effectif de $16 \mathrm{~h}$ par jour. Les animaux sont nourris ad libitum avec un aliment concentré complet du commerce.

La saillie post partum est réalisée sur des femelles, toutes primipares, $24 \mathrm{~h}$ à $36 \mathrm{~h}$ après la parturition afin de profiter simultanément de l'état d'œstrus et d'une certaine involution de l'utérus. Dans la même période des adoptions de lapereaux ont parfois été réaliśé pour que le nombre de petits allaités soit au moins de 4 afin de maintenir un état de lactation "normal ". Seules Ir femelles sur les 273 étudiées, réparties aléatoirement selon les génotypes, n'allaitaient plus au moment de la laparotomie. Celle-ci est effectuée 16 jours après la saillie et le tractus génital est prélevé.

\section{3. - Les caractères mesurés et leur signification biologique}

Pour chacune des femelles, deux types d'information ont été enregistrés. - A la première mise-bas :

le nombre de saillies pour obtenir la mise-bas (les femelles non gestantes après 3 saillies consécutives sont éliminées),

le nombre de lapereaux nés totaux et nés vivants,

le poids des femelles à la saillie post partum. 
- Après 1a laparotomie :

1a présence de corps jaunes, indicateur tout ou rien de l'ovulation,

le nombre d'ovules pondus (mesurés par le nombre de corps jaunes),

le nombre de sites d'implantation,

le nombre d'embryons,

le poids des embryons (analysés ultérieurement).

A partir de ces informations il est possible de définir les différents paramètres biologiques concernant la fécondité d'une femelle.

- La fertilité représente l'aptitude des femelles d'une souche donnée à faire le plus grand nombre possible de portées. Ce phénomène est concrétisé généralement par un taux de mise-bas : nombre de femelles mettant bas/nombre de femelles saillies. Ce taux résulte notamment du taux de femelles ovulant : nombre de femelles ovulant/nombre de femelles saillies. Nous définirons de plus le taux de gestation à 1 'instant $t$ par le rapport : nombre de femelles ayant au moins I embryon à l'instant $t /$ nombre de femelles saillies.

Le nombre de femelles gestant est parfois rapporté au nombre de femelles ovulant, donnée qui permet d'illustrer plus concrètement le pourcentage de femelles ovulant ne présentant pas de développement embryonnaire.

- La prolificité représente la taille de portée (nombre moyen de nés totaux et de nés vivants par rapport aux femelles mettant bas). Nous en avons analysé les composantes (moyennes d'ovules pondus, de sites, d'embryons) établies sur la population des femelles ovulant, ce qui permet de tenir compte de l'effectif complet des ovules n'ayant pas donné naissance à un produit. Ces moyennes peuvent être également calculées sur la population donnant lieu à l'événement, respectivement les femelles implantant et gestant à $\mathbf{r} 6$ jours, ce qui permet d'estimer la taille de portée potentielle. Les analyses de corrélation ont été effectuées dans cette optique.

La fréquence des pertes d'ovules englobe ceux non fécondés et ceux donnant naissance à un produit létal. Il est possible d'envisager cet événement sous deux formes :

La population des femelles ovulant fournit globalement un pool de corps jaunes, de sites et d'embryons. La fréquence des pertes avant implantation est représentée par la formule : (nombre de corps jaunes-nombre de sites)/nombre de corps jaunes, et après implantation : (nombre de sites-nombre d'embryons) / nombre de sites. Dans notre expérience, le premier type de pertes est certainement un peu surestimé, les sites porteurs d'embryons dégénérant dans les $48 \mathrm{~h}$. après leur implantation n'étant peut-être plus visibles à I 6 jours bien que ALLEN et al. indiquent que, en tuant expérimentalement tous les embryons à ro jours I/2, les sites sont encore visibles à $\mathrm{I} 7$ jours $\mathrm{I} / 2$.

Par ailleurs, il est intéressant de distinguer parmi les femelles ovulant, celles ne présentant aucun développement embryonnaire (o site ou o embryon considérés comme des pertes totales), de celles qui possèdent des embryons (pertes partielles), les causes de ces mortalités pouvant être éventuellement disjointes.

\section{4. - Les modèles d'analyse et la signification génétique des estimées}

\section{I. Les modèles d'analyses}

Les données ont été analysées suivant la méthode des moindres carrés (HARVEY, I960) avec test et estimation des effets considérés comme fixés et dans un schéma croisé. 
Le modèle général s'écrit :

$$
\mathrm{X}_{i j k}=\mu+a_{i}+b_{j}+(a b)_{i j}+e_{i j k}
$$

où $\mathrm{X}_{i j k}$ est la mesure faite sur la $k^{\text {eme }}$ femelle issue de la $i$ eme race de père et de la $j$ eme race de mère.

Une analyse de covariance a aussi été effectuée sur certaines données selon le modèle suivant d'analyse :

$$
\mathrm{Y}_{i j k}=\mu+a_{i}+b_{j}+(a b)_{i j}+\beta \mathrm{X}_{i j k}+e_{i j k}
$$

où $\mathrm{Y}_{i j k}$ est la mesure faite sur la $k^{\mathrm{eme}}$ femelle issue de la $i$ eme race de père et le $j$ eme race de mère et où $\mathrm{X}_{i j k}$ est la mesure faite sur la même femelle pour un autre caractère, exprimé plus tôt dans le cycle de reproduction. Ce modèle permet par exemple d'estimer les effets des différentes souches, pour le nombre de sites d'implantation linéairement indépendamment du nombre de corps jaunes.

Pour faire l'analyse de variance des pourcentages selon un modèle à effets fixés à deux facteurs sans interaction la méthode de GABRIEL (I963) a été utilisée.

Les calculs ont été réalisés d'une part sur l'ordinateur I.B.M. 370-I45 du Centre de traitement de l'information de Jouy-en-Josas et d'autre part, sur 1'ordinateur MITRA I5-35 du Centre de traitement de l'information de Toulouse.

\subsection{La signification des estimées}

L'analyse génétique est conduite en attribuant conventionnellement des variables à la mère ou au produit (fig. I).

- La présence (ou 1'absence) de corps jaunes (exprimée par la fréquence des femelles ovulant), et le nombre de corps jaunes sont considérés comme des caractères appartenant à la femelle (génération $n$ ).

L'obtention ou non de gestation suivie de parturition (fréquence des femelles mettant bas) est un phénomène plus complexe puisqu'il dépend du nombre de femelles ovulant, de leur fécondation puis du développement d'au moins I embryon. Le premier événement étant de loin déterminant, le taux de mise-bas sera attribué à la femelle.

Les résultats portent sur les valeurs moyennes entre génotypes et sur les effets d'bétérosis direct par comparaison entre les performances des femelles de souche pure et croisées. Les effets génétiques directs sont estimés en considérant les performances des 3 races de père sur leurs filles. La comparaison des effets race de père et race de mère de la femelle fournit une estimation de l'effet maternel de chacune des races mises en comparaison.

- Les informations concernant des caractères biologiques postérieurs à la fécondation (nombre de sites et d'embryons, taille de portée) sont considérés comme dépendant du produit donc appartenant à la génération $n+\mathrm{I}$. En effet, ces effectifs dépendent entre autre de la viabilité intrinsèque de chaque œuf.

Il convient alors d'envisager les origines paternelles et maternelles de ce produit. Les pères étant d'une souche unique et différente, l'analyse permettra seulement de démontrer l'existence éventuelle d'un effet du mâle sur la taille de sa portée.

En ce qui concerne la mère, les informations sont du même type que celles évoquées ci-dessus avec une génération de décalage. Dans cette optique, la valeur moyenne d'un génotype rend compte des effets globaux d'une femelle sur les 


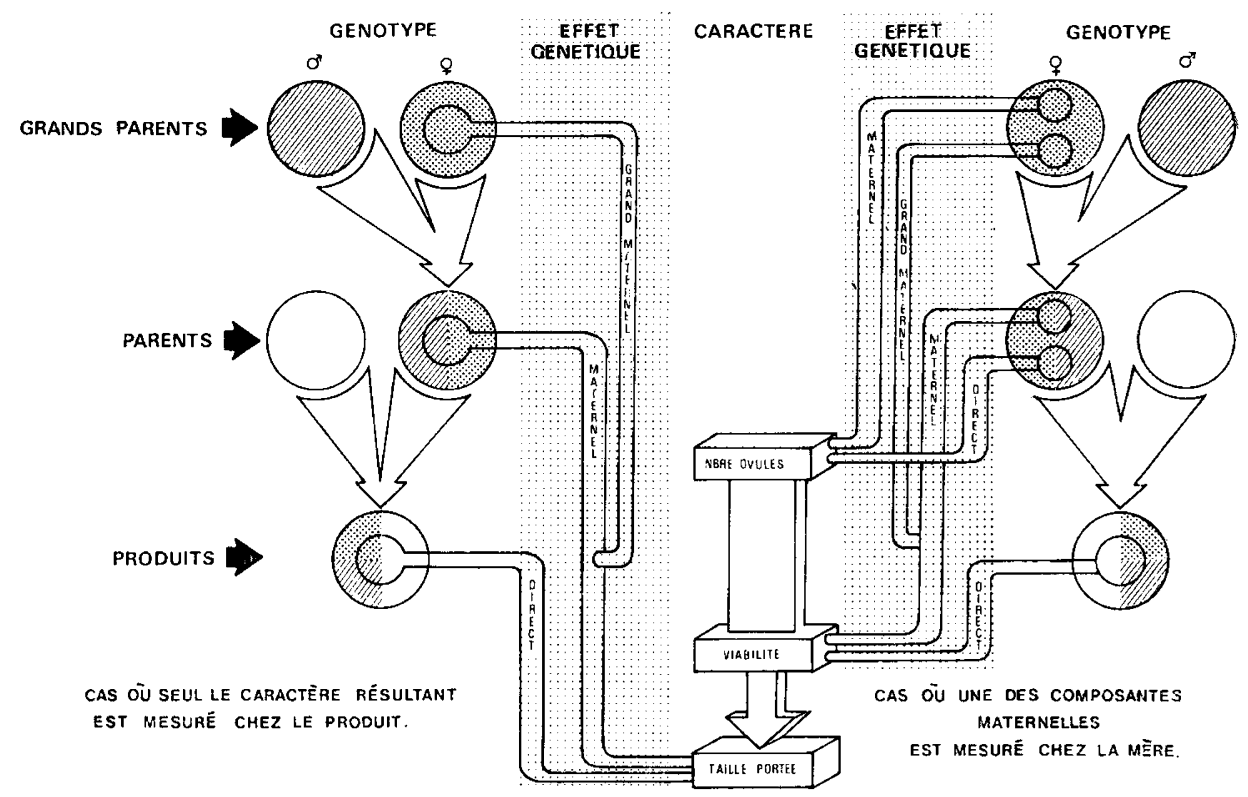

FIG. $\mathrm{x}$. - Les effets génétiques directs et indirects sur la taille de portée ou ses composantes. Direct and indirect genetical effect on the litter size or on its components

caractères mesurés chez son descendant : effets directs, maternels, grands-maternels et d'hétérosis maternel. L'effet race de père de la femelle inclut les effets génétiques directs et maternels (fig. I). Enfir la différence entre les effets race de mère et race de père de la femelle est une estimation de l'effet grand-maternel sur le caractère mesuré chez son ascendant.

\section{Résultats}

Les résultats traitant de l'influence du père de portée I et 2 sont tout d'abord brièvement exposés. Les données concernant les caractères liés au taux de mise-bas et à ses composantes sont ensuite regroupées. Celles se rapportant à la prolificité (taille de portée I) puis à ses composantes (nombre d'ovules pondus et mortalité embryonnaire) sont alors envisagés. Enfin un tableau de corrélation indique les liaisons éventuelles entre les caractères des portées I et 2.

\section{I. - Effet du père de la portée}

Le tableau 2 indique les effets du père de la portée sur les différentes variables mesurées et leur interaction éventuelle avec le type génétique de la femelle accouplée. Le mâle a une influence significative sur le nombre de sites et d'embryons. Cet effet est une combinaison du pouvoir fécondant du sperme et de la viabilité des embryons résultant des gènes transmis par ce mâle. L'absence d'interaction permet de considérer l'effet du père de la portée comme indépendant du génotype des femelles auquel il est accouplé, aussi ne sera-t-il pas pris en compte dans la suite de l'analyse relative aux génotypes maternels. 
TA,BLEAU 2

Effets du mâle, du type génétique de la temelle accouplée et de leur interaction sur les caractères mesurés: Valeurs et signification du $F$

\begin{tabular}{|c|c|c|c|c|c|}
\hline \multirow[b]{2}{*}{ Effets } & \multicolumn{2}{|c|}{ Portée I } & \multicolumn{3}{|c|}{ Portée 2} \\
\hline & $\begin{array}{l}\text { Nés } \\
\text { totaux }\end{array}$ & $\begin{array}{c}\text { Nés } \\
\text { vivarits }\end{array}$ & $\begin{array}{c}\text { Nombre } \\
\text { corps jaunes }\end{array}$ & $\begin{array}{l}\text { Nombre } \\
\text { de sites }\end{array}$ & $\begin{array}{l}\text { Nombre } \\
\text { d'embryons }\end{array}$ \\
\hline $\begin{array}{l}\text { Père de la portée (Fauve } \\
\text { de Bourgogne). }\end{array}$ & $0,549 \mathrm{NS}$ & $0,728 \mathrm{NS}$ & $\mathrm{I}, 480 \mathrm{NS}$ & $2,327\left(^{* *}\right)$ & $2,300(* *)$ \\
\hline $\begin{array}{l}\text { Type génétique de la femelle } \\
\text { accouplée }\end{array}$ & $\mathrm{r}, 346 \mathrm{NS}$ & $2,692\left({ }^{*}\right)$ & $8,028(* *)$ & $4,243\left({ }^{* *}\right)$ & $\left.3,219{ }^{* *}\right)$ \\
\hline Interaction & $0,998 \mathrm{NS}$ & $0,974 \mathrm{NS}$ & $\mathrm{r}, 3 \mathrm{r}_{5} \mathrm{NS}$ & $\mathrm{I}, 204 \mathrm{NS}$ & $\mathrm{I}, 090 \mathrm{NS}$ \\
\hline
\end{tabular}

NS : Non significatif.

${ }^{*}$ ) Significatif au seuil 5 p. roo.

(*) Significatif au seuil I p. roo.

\section{2. - Fertilité: Caractères liés au taux de mise-bas et à ses composantes}

Le tableau 3 donne la répartition par génotype de la fréquence des saillies fécondantes chez les femelles nullipares indiquant une moyenne générale de 66 p. Ioo (amplitude 58 à 75 p. I0o), ce qui correspond à I,5 saillie pour obtenir une mise-bas. Les valeurs de $\chi^{2}$ indiquent que le génotype de la femelle (au seuil de I p. Ioo) et que le croisement (au seuil de 4 p. Ioo) interviennent sur cette fréquence. En définitive les animaux ayant des gènes Petits Russes paraissent un peu plus fertiles, et l'ensemble des femelles croisées bénéficie par rapport à celles de races pures, d'un effet d'hétérosis de l'ordre de I3 p. Ioo.

L'incidence des types génétiques paternels et maternels des femelles sur la fréquence des saillies fécondantes est faible (tabl. 3). Les effets race de mère, bien que non significatifs, semblent supérieurs aux effets race de père. La race Petite Russe paraît transmettre les meilleures aptitudes, les filles issues de cette origine maternelle présentant une supériorité de 5 p. Ioo par rapport aux deux autres origines.

Le tableau 4 indique les taux de femelles ovulant en saillie post partum selon le génotype. Pour une valeur moyenne de $83,20 \mathrm{p}$. Ioo existe une variation allant de Ioo p. Ioo (génotype Petit Russe) à $6 \mathrm{I}, 8$ p. Ioo (génotype Néo-Zélandais). Cette différence entre génotypes très significative $\left(\chi^{2}<\mathrm{I}\right.$ p. Ioo) provient essentiellement de la meilleure aptitude à l'ovulation des femelles Petites Russes et métisses Petites Russes - 88,82 p. 100 - par rapport aux femelles des autres génotypes $-76,02$ p. roo - $\left(\chi^{2}<\right.$ I p. roo). La supériorité du lot de femelles croisées $-85,22$ p. IOopar rapport à celui des femelles de souches pures - 77,I4 p. I00 - n'intervient qu'à un moindre degré (hétérosis de 9 p. roo). 
TABLEAU 3

Fréquence des saillies fécondantes dans les 9 génotypes de femelles nullipares Estimées des effets race de père et vace de mère

\begin{tabular}{|c|c|c|c|c|}
\hline Race mère & $\mathrm{CA}$ & $\mathrm{NZ}$ & PR & $\begin{array}{l}\text { Estimée des effets } \\
\text { race de mère } \\
\mathrm{F}=0,525 \mathrm{NS}\end{array}$ \\
\hline $\mathrm{CA} \ldots \ldots \ldots \ldots$ & 65,7 & 65,8 & 63,2 & $-5,8$ \\
\hline $\mathrm{NZ}$. & 60,6 & $5^{8,6}$ & 75,0 & $-4,6$ \\
\hline $\mathrm{PR} \ldots .$. & 70,6 & 75,5 & $59, \mathrm{r}$ & 0,0 \\
\hline $\begin{array}{c}\text { Estimée des effets race de père }(\mathrm{F}= \\
0,064 \mathrm{NS})\end{array}$ & $-\mathrm{r}, \mathbf{I}$ & $-2,7$ & 0,0 & $\left(^{*}\right) 66,4$ \\
\hline
\end{tabular}

Population constituée de femelles ayant réalisé une mise bas après $\mathbf{I}, 2$ ou 3 saillies.

Les fréquences sont exprimées en p. roo. (*) Fréquence portant sur l'ensemble des femelles.

Ies effets race de père et race de mère sont estimées par la méthode de GabRIEL (I963).

\section{TABLEAU 4}

Fréquence des femelles primipares ovulant dans les 9 génotypos en saillies Post-Partum Estimées des effets race de père et race de mère

\begin{tabular}{|c|c|c|c|c|}
\hline Race mère & CA & $\mathrm{N} Z$ & PR & $\begin{array}{l}\text { Estimées des effets } \\
\text { race de mère } \\
\mathrm{F}=1,75^{2} \mathrm{NS}\end{array}$ \\
\hline . . . . . . . . & 87,0 & 85,2 & 70,8 & $-12,6$ \\
\hline $\mathrm{NZ}$ & 75,7 & $6 \mathrm{I}, 8$ & 92,3 & $-\mathrm{I} 6,8$ \\
\hline $\mathrm{PR} \cdot \cdot \cdot \cdot$ & $9^{\mathrm{I}, 7}$ & 90,0 & IOO,O & 0,0 \\
\hline $\begin{array}{l}\text { Estimées des effets race de père }(\mathrm{F}= \\
0.657 \mathrm{NS})\end{array}$ & $-5,2$ & - I I,I & 0,0 & $\left.{ }^{*}\right) 8_{3}, 2$ \\
\hline
\end{tabular}

Femelles du tableau 3, soumises à une saillie unique.

Les fréquences sont exprimées en p. roo. $\left(^{*}\right)$ Fréquence portant sur l'ensemble des femelles.

I,es effets race de père et race de mère sont estimés par la méthode de Gabriel (I963). 
Les origines génétiques paternelles et maternelles des femelles saillies n'ont que peu d'incidence sur leur aptitude à ovuler.Les effets race de mère, à la limite de la signification au seuil de $25 \mathrm{p}$. Ioo, semblent supérieurs aux effets race de père et les origines Petites Russes sont là encore les meilleures pour ce caractère.

Le taux de gestation est très proche du taux d'ovulation cependant près de ro p. roo des femelles ovulant n'ont pas de sites d'implantation ou d'embryons à 16 jours.

\section{3. - Prolificité en portée I}

Le tableau 5 indique les moyennes générales du nombre de nés totaux $(7,95 \pm$ $0,22)$ et de nés vivants $(7,43 \pm 0,24)$ chez l'ensemble des femelles nullipares ainsi que les écarts à ces moyennes pour chacun des génotypes. L'effet du génotype n'est significatif que sur le nombre de nés vivants, dî̀ en grande part aux très mauvaises performances de la femelle Petite Russe. Le croisement améliore la taille de portée (tabl. 6) : l'hétérosis maternel global est de $6,5 \mathrm{p}$. Ioo pour le nombre de nés totaux et de $\mathrm{I} 2,4 \mathrm{p}$. Ioo pour les nés vivants. Il est surtout élevé pour les souches associées au Petit Russe. La combinaison entre le Néo-Zélandais blanc et le Californien, bien qu'étant à la base du croisement donnant la meilleure performance, ne fournit pas d'hétérosis maternel.

Le tableau 6 fournit les estimées des effets race de père et race de mère des femelles sur la taille de leur portée (nés totaux et nés vivants). L'effet race de père est seul significatif aux seuils de $5 \mathrm{p}$. Ioo et $\mathrm{I}$ p. Ioo respectivement avec de plus interaction avec la race de mère pour le nombre de nés vivants. Le père NéoZélandais a un effet positif de 0,33 et 0,56 lapereaux sur les deux caractères (nés totaux, nés vivants) alors que celui du père Petit Russe est très défavorable $(-0,47$ et - 0,63$)$. La mère Petite Russe a également une incidence très négative. L'in-

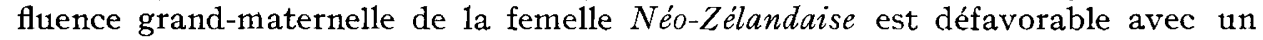
effet significatif pour le nombre de nés vivants.

\section{4. - Composantes de la prolificité en portée 2}

\section{I Nombre d'ovules pondus}

Le nombre moyen de corps jaunes pour les 227 femelles ovulant est de $10,23 \pm$ 0,20 (tab1. 5) avec une amplitude comprise entre 4 et I6. Une variation de II à 8,5 corps jaur.es, significative au seuil de I p. Ioo est enregistrée entre les moyennes par génotype.

La figure 2 illustre le classement des génotypes sur ce caractère : les femelles ayant des origines Californiennes ou Néo-Zélandaises ont les valeurs les plus élevées. L'hétérosis direct global est très significatif $(9,23 \mathrm{p}$. roo) provenant surtout de la supériorité des femelles croisées Californiennes-Néo-Zélandaises ( + I2,8I p. Ioo).

Le tableau 6 indique que l'origine génétique des parents a un effet significatif sur le nombre d'ovules pondus par leurs filles. La souche Californienne en tant qu'origine paternelle $\in t$ maternelle transmet aux descendants $u n$ bon niveau d'ovulation ( $+0,69$ et $+0,9$ I corps jaunes respectivement), la souche Petite Russe engendre une faible ponte $1-0,5$ I et - 0,87 corps jaunes). Les effets maternels sont dans tous les cas faibles. 


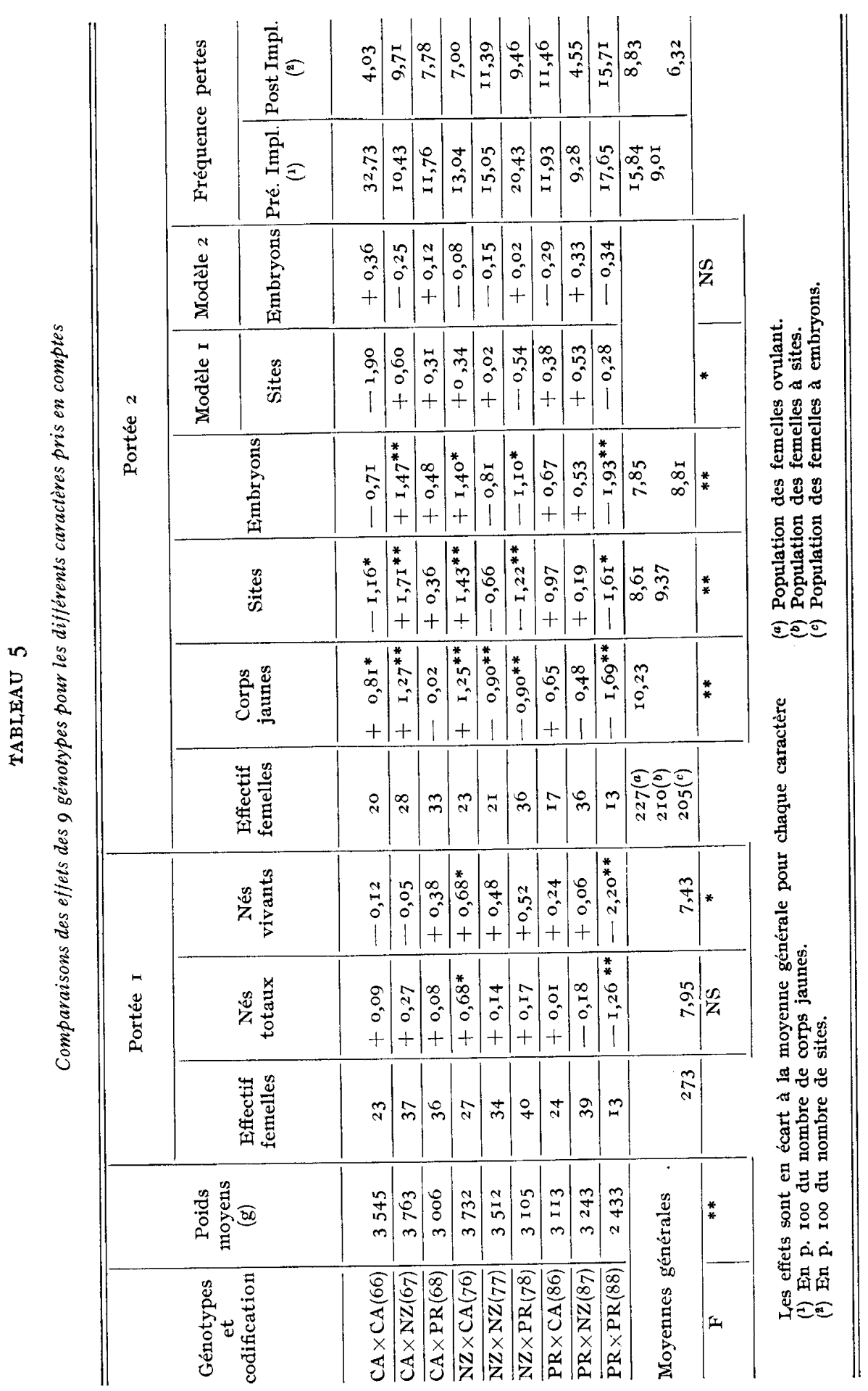




\subsection{Mortalité embryonnaire}

\subsection{Nombre de sites d'implantation et d'embryons observés}

Ces résultats sont rapportés aux tableaux 5 et 6 . Dans la population des femelles ovulant, le nombre moyen de sites est de $8,6 \mathrm{I} \pm 0,34$, celui des embryons de $7,85 \pm 0,37$, les valeurs extrêmes allant de 0 à $I_{5}$ pour les deux caractères (les nombres moyens de sites et d'embryons rapportés à la population des femelles concernées par l'événement sont également mentionnés : 9,37 et 8,8I). Les moyennes par génotypes : 7,00 à I0,32 pour les sites, 5,92 à 9,32 pour les embryons diffèrent de façon hautement significative.
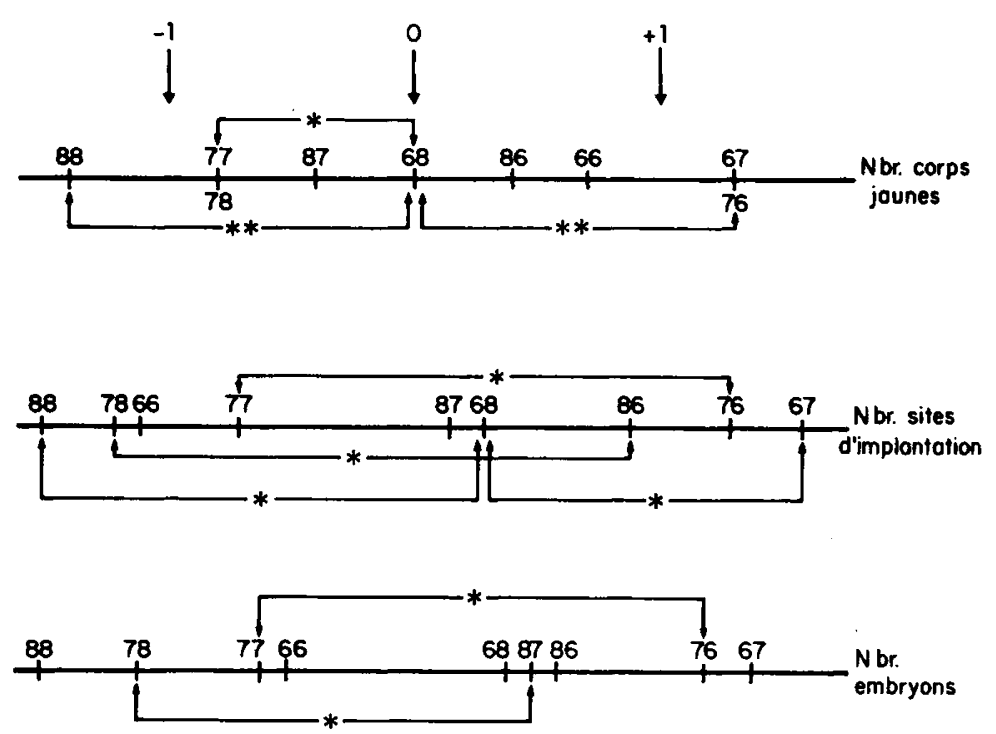

FIG. 2. - Classement des génotypes selon le nombre de corps jaunes, de sites et d'embryons

Classification of genotypes according the number of yellow bodies of sites and of embryos

De même que pour le nombre de corps jaunes, le classement par génotype (fig. 2) fait apparaître la supériorité des génotypes croisés Californiens-NéoZélandais et la faible prolificité de la femelle Petite Russe. Par contre, la souche Californienne a un rang sur le nombre d'implants et de fœtus très inférieur à celui relatif à 1'ovulation. L'hétérosis maternel global est très significatif I9,80 p. Ioo et $23, \mathrm{I} 2 \mathrm{p}$. Ioo. Il est essentiellement dû au croisement de la souche Californienne avec les deux autres.

L'incidence des origines génétiques paternelles et maternelles est comparable sur le nombre des sites et des embryons. Seule la race de mère a une influence sur la taille de portée potentielle de ses filles; celle-ci est due à l'effet très défavorable de la souche Petite Russe ( $-0,8 \hat{j}$ site et - 0,85 embryon) qui semble luimême lié à un effet grand-maternel également négatif. L'effet grand-maternel de

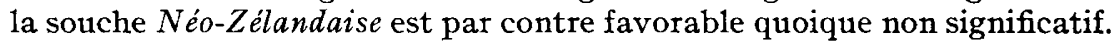




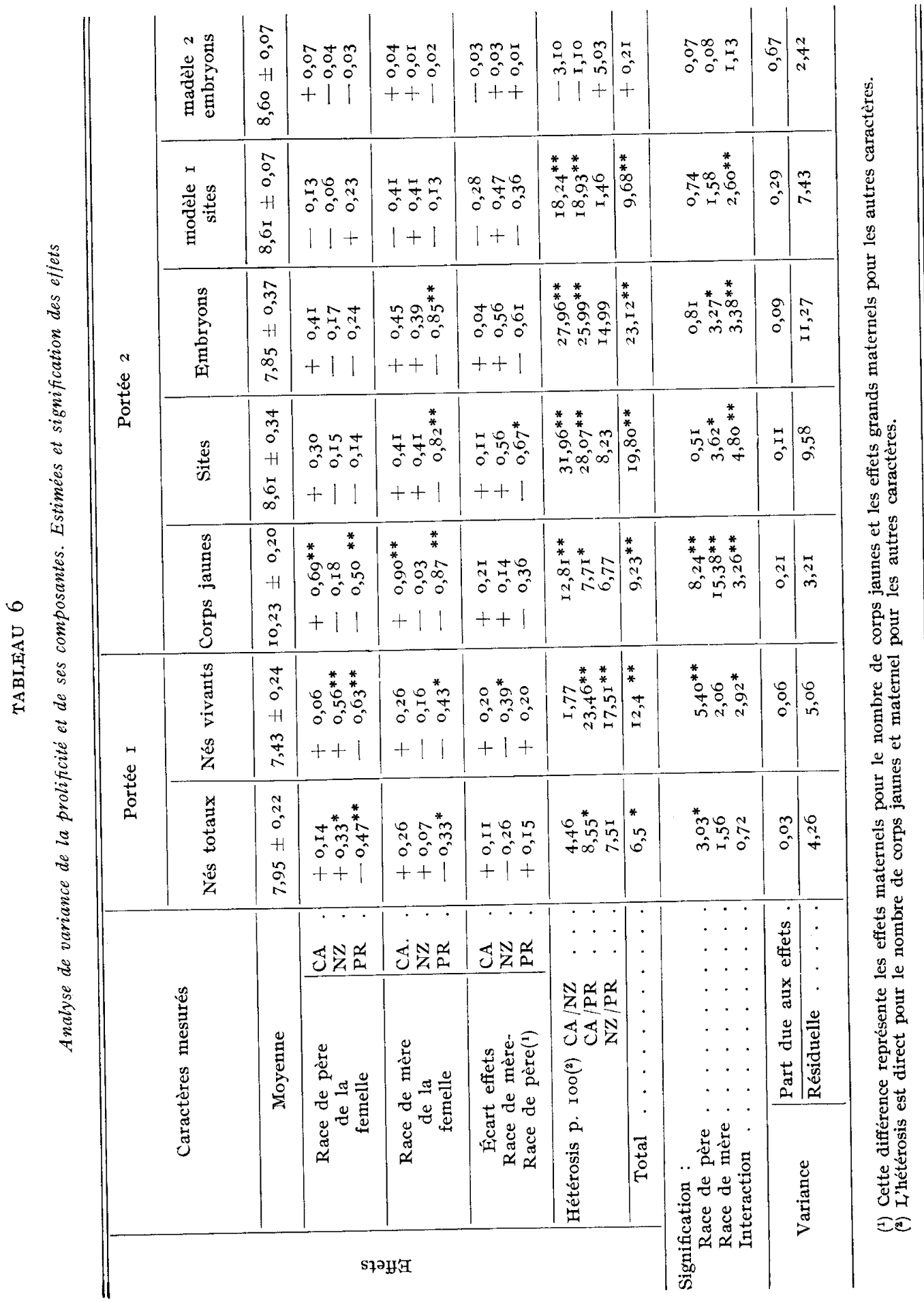




\subsection{Nombre de sites d'implantation et d'embryons calculés}

Les nombres de sites et d'embryons mesurés sont largement conditionnés par la variable qui leur a donné naissance : le nombre d'ovules pondus. Aussi apparaît-il important pour mieux appréhender les différences réelles entre génotypes liées à la seule viabilité de faire abstraction des effectifs de départ en introduisant ceux comme covariables dans les modèles d'analyse.

Les tableaux 5 et 6 indiquent les nouvelles significations des différents effets génétiques sur le nombre de sites et d'embryons en prenant respectivement comme covariable le nombre de corps jaunes (modèle I) puis le nombre de sites (modèle 2 ). Des différences significatives n'apparaissent alors entre génotypes que pour le nombre de sites. Les femelles croisées bénéficient d'un effet d'hétérosis d'environ Io p. Ioo dû au croisement de la souche Californienne avec les deux autres. Les

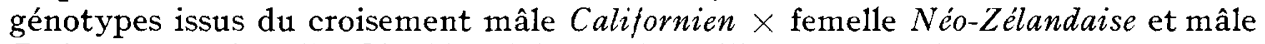
Petit Russe $\times$ femelle Néo-Zélandaise ont la meilleure viabilité.

Les effets race de père ne sont pas significatifs : toutefois la souche Petite Russe semble transmettre une bonne aptitude à implanter un nombre d'œufs donnés ( $+0,23$ sites) mais l'effet grand maternel est défavorable. La souche Néo-Zélandaise blanche paraît être la meilleure race de mère pour la viabilité préimplantatoire, aptitude liée à un effet grand maternel lui-même positif.

Les effets génétiques n'ont que peu d'incidence sur le nombre d'embryons, à nombre de sites fixés.

\subsection{Fréquence des pertes embryonnaires}

Parmi les 227 femelles ovulant, la fréquence des pertes avant implantation est de I $_{5}, 84$ p. Ioo (tab1. 5). Des différences hautement significatives $\left(\chi^{2}<\right.$ I. p. IOO) sont enregistrées entre les génotypes qui présentent des valeurs extrêmes allant de 9,28 p. Ioo à $32,73 \mathrm{p}$. IOo. Les génotypes croisés manifestent une meilleure viabilité que les souches pures, l'hétérosis maternel étant de l'ordre de ro p. roo.

La mortalité après implantation est de 8,83 p. Ioo à I 6 jours. Les différences entre génotypes (valeurs extrêmes allant de 4,05 à $\mathrm{I} 5,7 \mathrm{I}$ ) sont très significatives, $\chi^{2}<$ I p. Ioo, mais les femelles croisées ne présentent pas en moyenne de supériorité par rapport aux femelles de races pures.

Par ailleurs, il est possible de considér $€ \mathrm{r}$ que la population des femelles ovulant se compose de 2 ro femelles présentant des signes d'implantation et de $I 7$ femelles vides. Les pertes partielles pré-implantatoires s'élèvent alors à 9 p. roo, les valeurs extrêmes allant de 5,I7 à I3,33 p. roo. Les différences entre génotypes sont atténuées et les femelles croisées ne présentent plus de supériorité.

\section{5. - Liaison entre les caractères}

Les corrélations phénotypiques ont été calculées entre les caractères quantitatifs (effectifs) entre et intra génotype (tab1. 7). Aucun test ne permet de rejeter l'hypothèse de linéarité des liaisons observées entre ces caractères.

Pour chacune des deux mises-bas mesurées, la variabilité augmente lorsque, pour les caractères de productivité numérique, ceux-ci sont plus éloignés de la ponte ovulaire. Du fait de l'absence de variabilité, entre génotypes, pour le nombre de nés totaux, seules les corrélations intra-génotype avec ce caractère ont un sens. En première mise-bas la corrélation entre nés totaux et nés vivants est forte $(+0,9)$. 
TABLEAU 7

Corrélation phénotypique entre les caractères et leurs variances mesurés en mise bas $I$ et après la saillie Post-partum

\begin{tabular}{|c|c|c|c|c|c|c|}
\hline & $\begin{array}{l}\text { Nés } \\
\text { totaux }\end{array}$ & $\begin{array}{c}\text { Nés } \\
\text { vivants }\end{array}$ & $\begin{array}{l}\text { Nombre } \\
\text { corps } \\
\text { jaunes }\end{array}$ & $\begin{array}{l}\text { Nombre } \\
\text { de } \\
\text { sites }\end{array}$ & $\begin{array}{l}\text { Nombre } \\
\text { d'em- } \\
\text { bryons }\end{array}$ & $\begin{array}{l}\text { Poids } \\
\text { (g) }\end{array}$ \\
\hline Nés totaux & $\begin{array}{r}0,014 \\
4,25^{8}\end{array}$ & & & & & \\
\hline Nés vivants. . . . . & 0,87 & $\begin{array}{r}0,223 \\
5,060\end{array}$ & 0,49 & 0,67 & 0,79 & 0,60 \\
\hline Nombre corps jaunes . & 0,09 & 0,07 & $\begin{array}{r}0,84^{\circ} \\
3,209\end{array}$ & 0,86 & 0,93 & 0,77 \\
\hline Nombre de sites ... & $-0,04$ & $-0,05$ & 0,48 & $\begin{array}{r}0,988 \\
9,579\end{array}$ & $\mathrm{I}, 02$ & 0,64 \\
\hline Nombre d'embryons & $-0,04$ & $-0,01$ & 0,45 & 0,89 & $\begin{array}{r}0,776 \\
\text { I I , 265 }\end{array}$ & 0,73 \\
\hline Poids de la femelle (g) & 0,09 & 0,08 & 0,39 & 0,14 & 0,13 & $\begin{aligned} \text { I } 26863 \\
85 \text { 8о r }\end{aligned}$ \\
\hline
\end{tabular}

I.es valeurs situées au-dessus de la diagonale sont calculées entre génotypes. Celles figurant en-dessous sont calculées intra-génotype.

I.es variances (entre et intra génotype) figurent sur la diagonale principale.

Les caractères relatifs à la seconde gestation sont d'autant plus liés entre eux qu'ils sont d'expressions temporelles plus proches. Les valeurs élevées trouvées pour les corrélations entre génotypes rendent compte des différences mises en évidence plus haut (voir classement des génotypes au tableau 5 pour ces 3 caractères). Les corrélations calculées intra-génotype montrent que le nombre de corps jaunes est peu lié avec les autres composantes de la productivité numérique (ce dernier n'explique que $25 \mathrm{p}$. Ioo de la variabilité observée pour les deux autres caractères). Par contre, les nombres de sites et d'embryons sont phénotypiquement très liés $(+0,9)$. Notons au passage que ce résultat vient en faveur de la convention adoptée pour l'attribution à la mère ou au produit des caractères mesurés.

Le nombre de nés vivants est corrélé, entre génotypes, avec les caractères mesurés lors de la seconde gestation. La corrélation est d'autant plus forte que les caractères sont physiologiquement plus proches. Là encore, le nombre de corps jaunes n'explique que $25 \mathrm{p}$. 100 de la variabilité observée sur le nombre de nés vivants entre génotypes. Il n'y a pas de liaison intra-génotype entre les caractères de la première mise-bas et ceux de la seconde gestation. 
Les corrélations entre génotypes relatives aux caractères de prolificité et aux poids des femelles sont la conséquence de l'introduction de la race Petite Russe dans le schéma expérimental. En effet cette race de petit format se caractérise par une faible taille de portée (liée à un faible niveau ovulatoire). En conséquence, les caractères intervenant (poids et ovulation) étant surtout additifs, entre races, les corrélations observées sont fortes $(0,60$ à 0,80$)$. Intra-génotype cet effet s'annule et seule la corrélation entre le poids de la femelle et le nombre d'ovules pondus $(0,39)$ paraît être intéressante à considérer.

\section{Discussion}

Le lapin, animal de production et matériel expérimental, a fait l'objet de recherches très diversifiées. Les travaux physiologiques sur la reproduction sont relativement nombreux et nous nous appuierons sur ceux-ci pour discuter nos tésultats. Par contre, les recherches en génétique sont dans ce domaine limitées et déjà anciennes (répertoriées par RoBINSON, I958), limitant ainsi notre discussion à un essai de synthèse des résultats les plus marquants. En ce qui concerne 1'estimation des effets génétiques, dans tous les cas, nous devons remarquer la faible contribution du modèle interprétatif utilisé à la variabilité des mesures effectuées. On constate, néanmoins, que la variabilité résiduelle est d'autant plus diminuée que le caractère est plus analytique. C'est le cas du nombre de corps jaunes et de la viabilité des ovules fécondés. Les valeurs très faibles $\left(R^{2}\right.$ inférieur à 0,10$)$ pour les autres caractères fournissent de sérieuses limites quant à la généralisation des tendances rapportées ci-après et ci-dessus.

\section{I. - La Fertilité}

L'analyse des composantes du taux de mise-bas confirme que celui-ci est largement conditionné par l'aptitude des femelles à ovuler. Il est bien connu que la ponte ovulaire peut ne pas avoir lieu à la suite du coït (SAWIN, I954; Foote et al., r963; Fox, I968) avec une fréquence qui varie dans de très larges proportions.

Lorsque l'ovulation se produit, les 7 p. Ioo de femelles vides observées au moment de l'implantation (avec une amplitude de o à $25 \mathrm{p}$. Ioo selon les génotypes) se situent dans l'ordre de grandeur des valeurs généralement annoncées en littérature : 5,2 p. 100 selon BRAMBel, (r948), 4,8 p. Ioo selon ADAMs (rg6o $a$ et $b$ ), 9 à I4 p. IOo selon SELME et PRUD'Hon (I973), I3 p. IOo selon Hulot et POUJARDIEU (I976) en insémination artificielle. L'absence complète de sites d'implantation peut être due à une mortalité embryonnaire précoce ou à un défaut de fécondation, éventualité que ADAMs (r965) chiffre aux environs de $5 \mathrm{p}$. Ioo.

En dépit de différences importantes concernant la parité des femelles d'une part et l'obtention du taux de mise-bas et du taux de femelles ovulant d'autre part, des tendances communes se dégagent à partir de ces deux types de résultats : les femelles croisées ont une fertilité supérieure à celles des races pures (I3 p. Ioo). Elle peut être due à un certain hétérosis (9 p. I0o) sur l'aptitude à ovuler mais également au fait que les femelles croisées sont plus aptes à maintenir la gestation : dans l'expérience de transfert d'œufs rapportés par VENGE (I950) celles-ci ont en effet un taux de gestation de 69 , r p. Ioo contre $53,6 \mathrm{p}$. Ioo chez les femelles de races pures. 
Les femelles Petites Russes et métisses Petites Russes paraissent plus fertiles (malgré le taux de gestation un peu faible de $59 \mathrm{p}$. Ioo en mise-bas $\mathrm{I}$ ). Ces résultats rejoignent ceux de POUJARDIEU et VRILLON (I973) extraits d'un schéma expérimental identique qui trouve pour ces génotypes une supériorité de l'ordre de I3 p. roo. Ces informations confirment l'idée selon laquelle les souches de petite taille seraient plus fertiles que celles de grande taille : 4I,3 p. Ioo pour la Polonaise et 35,6 p. Ioo pour la Géante des Flandres selon VENGE (I950), 69 p. Ioo pour les Petite Russe et Dutch et 54 p. Ioo pour les Néo-Zélandaise et Californienne selon HuLOT (I975). Les mêmes constatations sur ces deux groupes de races se retrouvent en insémination artificielle chez des femelles nullipares ( 78 p. roo contre 62 p. Ioo) et primipares (50 p. IOO contre 35 p. IOO), selon HULOT (I975).

En définitive, la souche Petite Russe paraît de façon assez constante avoir une influence favorable sur la fertilité bien que l'estimation des effets génétiques directs soit faible.

\section{2. - Prolificité en mise-bas $I$}

Depuis Pickard (I930) jusqu'à Rouvier et al. (I973) il est reconnu que la première portée a un effet dépressif sur le nombre de nés totaux et nés vivants chez le lapin. Il est possible que cette expérience conduise aux mêmes constatations, la taille moyenne potentielle de la portée 2 étant de 8,8I lapereaux contre 7,95 nés totaux. Comme l'indiquent les tableaux 2 et 5 les effets génotype de la femelle sont peu ou pas significatifs sur ces valeurs. De plus, la variabilité observée pour le nombre de nés totaux est presque nulle. Nous ne pouvons en conséquence accorder une importance trop grande aux conclusions relatives à ces mesures.

Classiquement, les expériences portant sur la comparaison des tailles de portée à la naissance de deux races de formats différents montrent que plus la race est lourde, plus la taille de portée est grande : GREGORY (1932) trouve en comparant le Polonais au Géant des Flandres des valeurs respectives de 3,24 et Io, I 7 lapereaux. Rosahn et al. (1934), pour le Polonais et le Beveren observent 3,92 et 7, I4 lapereaux respectivement. Cette liaison poids-taille de portée a d'ailleurs aussi été observée intra race (ROSAHN et al., I934); VENGE (I950) pense lui, que la liaison intra-race est curvilinéaire car pour un des types génétiques de gros format il trouve une baisse de la taille de portée avec une augmentation de poids. Dans notre expérience les femelles de plus petit format produisent moins que les femelles de formats plus grands $(5,23$ pour la Petite Russe contre 7,9I pour la Néo-Zélandaise Blanche). Entre race, existe une corrélation de 0,60 entre le poids de la femelle à la saillie post partum et la taille de portée (nés vivants). Intra-race les conclusions de VENGE semblent confirmées car la liaison linéaire s'estompe (corrélation de o,08).

Des résultats portant sur les mêmes génotypes rapportés par MATHERoN et POUJARDIEU (r976) et MATHERon et RouviER (à paraître) confirment les valeurs observées dans cette expérience : les femelles croisées présentent ici une supériorité de 6 à I 3 p. Ioo (de Io à I $4 \mathrm{p}$. Ioo chez les auteurs cités ci-dessus) et le croisement met une femelle ayant des gènes Petits Russes au niveau de la femelle du génotype associé (complémentarité et hétérosis sur les composantes de la taille de portée).

Au total l'opposition, limitée à la portée I, qui apparaît entre effet de la race de père et effet grand-maternel (estimées du tab1. 6) pourrait être responsable du faible nombre d'effets " génotype femelle " significatifs. Cette disjonction entre deux performances successives d'une même femelle doit être pondérée par la faible contribution du modèle $\left(\mathrm{R}^{2}\right.$ compris entre 0,03 et 0,09$)$ et par les variances résiduelles qui varient du simple au double. 


\section{3. - Composantes de la prolificité en mise-bas 2}

\subsection{Le nombre d'ovules}

Les résultats de Gregory (I932) et de Venge (I950) sur le Polonais et le Géant des Flandres indiquent un nombre moyen d'ovules pondus de 3,97 et I2,88 pour le premier auteur, de 5,52 et 10,73 pour le second. L'étude de ces 2 seules souches extrêmes par leur format (poids moyen I $508 \mathrm{~g}$ et $5589 \mathrm{~g}$ selon GREGORY) ont conduit à admettre que plus la race est lourde, plus elle ovule et à établir pour chacune d'elle, un indice de prolificité potentielle correspondant à un ovule pour $x \mathrm{~g}$ de poids de corps. Gregory indique, pour la souche de petit format, I ovule pour $380 \mathrm{~g}$ et, pour celle de grand format, I ovule pour $434 \mathrm{~g}$; VENGE note des chiffres inférieurs : I ovule pour $297 \mathrm{~g}$ et $394 \mathrm{~g}$ respectivement. Nos souches présentent des écarts de format sensiblement plus faibles et des différences d'ovulation moindres ( 3 ovules maximum). La corrélation entre souche de 0,77 entre le poids de la femelle à la saillie post partum et le nombre de corps jaunes indique que dans de nombreux cas, plus la race est de grand format, plus elle ovule. En souches pures, la Petite Russe possède 8,5 corps jaunes, alors que la Californienne en a II. Toutefois, il est à noter que la souche Néo-Zélandaise d'un poids équivalent à la Californienne, au stade étudié, a un nombre significativement inférieur $(9, \mathrm{I})$ à ce qui serait attendu selon ces informations.

Intra race, VENGE (I950) observe les mêmes tendances que pour la taille de portée : le nombre de corps jaunes augmente avec le poids chez le Polonais alors qu'il diminue chez le Géant des Flandres. La corrélation intra-souche obtenue à partir de nos résultats $(0,39)$ est importante mais plus faible que celle enregistrée entre races. Cela peut suggérer que 1'amplitude des liaisons trouvées entre races était plus ou moins induite par l'introduction dans l'expérience de la race Petite Russe, et que la liaison poids-ovulation pourrait être curvilinéaire.

Le nombre d'ovules pondus chez le lapin est comme pour d'autres mammifères étudiés (souris, porc) soumis à des effets génétiques directs et c'est la seule composante de la prolificité où ils présentent un degré de signification.

Des effets maternels semblent apparaître (bien qu'ils ne soient pas significatifs), et vont dans le même sens que les effets directs. L'influence défavorable d'une mère Petite Russe peut avoir son origine dans le poids correspondant à ce type génétique. L'examen des portées dont sont issues les femelles mises en comparaison indique des valeurs allant dans le même sens que les poids des races de base $(6,46$ pour les issues de femelles Petites Russes, contre 8,24 et 8,07 pour les issues de femelles Néo-Zélandaises et Californiennes respectivement). L'origine des effets maternels ne peut donc clairement être reliée à l'une ou l'autre de ces mesures.

Enfin, il est important de souligner la part d'hétérosis non négligeable chez le lapin, contrairement à ce qui semble être rapporté par ailleurs ( 3 à 7 p. Ioo). La valeur de ro p. Ioo trouvée dans notre expérience paraît être un seuil minimal par rapport aux quelques résultats concernant la même espèce.

HAmmond (I928, I933, I934) trouve une valeur plus élevée de 23 p. Ioo (Io,4 corps jaunes pour le croisement entre deux souches ayant respectivement 5,8 et II,I corps jaunes moyens). GREGORY (1932) observe lui pour le croisement entre Polonais et Géant des Flandres une valeur moyenne de 7 , I corps jaunes (contre I 2,88 et 3,97 pour les races pures) soit une baisse par rapport à la moyenne des races pures de près de $\mathrm{r} 6 \mathrm{p}$. Ioo. Cela indique que chez le lapin, la ponte ovulaire n'a pas un déterminisme uniquement additif. 


\subsection{Liaison entre le nombre d'ovules pondus et le taux de femelles ovulant}

La question peut se poser de savoir si la fréquence d'ovulation (nombre de femelles ovulant pour roo femelles saillies) est lié au nombre d'ovules pondus. Entre race, la figure 3 nous indique la répartition des génotypes pour ces deux caractères. La courbe rend compte des observations faites jusqu'à présent sur le lien entre le poids d'une femelle et les deux caractères considérés; il apparaît alors un optimum résultant de l'aptitude des souches à se combiner. Intra génotype, nous avons réparti les femelles en deux lots. Un lot contenant les lapines dont les pleines sœurs ont donné o corps jaune, l'autre complémentaire. La moyenne du nombre d'ovules de chacun des deux lots est respectivement Io, II et 10,24. La différence, non significative, montre, intra-race, l'indépendance de ces deux caractères qui n'ont probablement pas le même déterminisme.

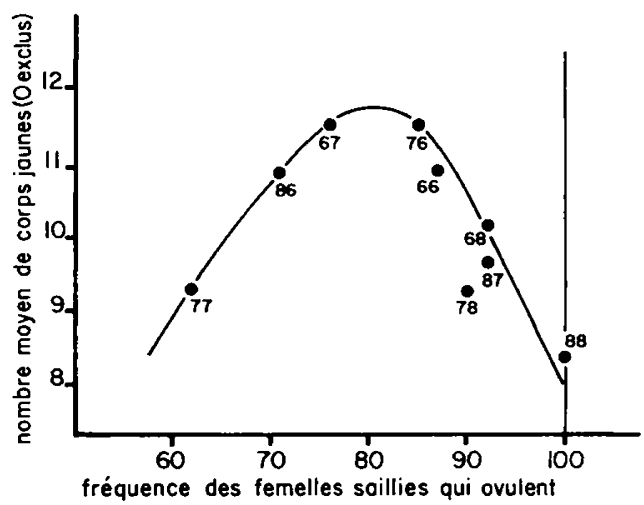

FIG. 3. - Liaison entre race, nombre de corps jaunes et fréquence des femelles saillies qui ovulent

Relationship between breed, number of yellow bodies and trequency of mated temales which leak

\subsection{Mortalité embryonnaire}

La différence entre le nombre d'ovules pondus et le nombre de fœetus viables est particulièrement importante chez le lapin : la mortalité globale est estimée à 43,3 p. Ioo chez l'animal sauvage selon BRAmbeL, (I948) et 30 p. Ioo chez le lapin domestique selon ADAMS (rg6oa).

Le niveau de mortalité préimplantatoire, concernant les pertes partielles paraît remarquablement constant lorsque nous comparons nos résultats : 9 p. Ioo, à ceux de BRAmbel, (I948) : Io-I3 p. roo et de AdAms (I96oa) : I I, 4 p. IOo (8 p. Ioo en portée 2 où les femelles présenteraient la meilleure viabilité selon cet auteur, I965). Lorsque les femelles ovulant vides sont inclues, nous obtenons un pourcentage de 15,84 p. IOO inférieur aux estimations de SELME et PRUD'HON ( 22 à 33 p. Ioo selon le rythme de reproduction : saillie 2 à 3 semaines après la mise-bas ou en post partum), ce qui paraît s'expliquer normalement par un pourcentage de femelles vides légèrement plus élevé ( 9 à I 4 p. Ioo chez ces auteurs) contre 7 p. Ioo dans notre expérience.

L'importance de la mortalité post-implantatoire paraît moins bien circonscrite, 
de l'ordre de $30 \mathrm{p}$. IOo pour BRAMBELL (I948) dont $70 \mathrm{p}$. IOo entre 9 et $\mathrm{r} 7$ jours, I 8,3 p. IOo selon ADAMS (I $960 a$ ) à 23 jours $(7 \mathrm{p}$. Ioo juste après l'implantation,

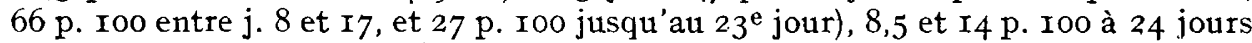
selon SELME et PRUD'HON (I973). Notre estimation à I6 jours de 6,3 p. roo paraît faible car à cette date, il semblerait d'après les 2 premiers auteurs, qu'environ les $2 / 3$ de la mortalité post implantatoire soit déjà atteint. Cette légère discordance dans les résultats pourraient provenir du fait que dans notre expérience 6 génotypes. sur 9 sont croisés (effet d'hétérosis maternels diminuant la mortalité post implantatoire observé par les auteurs en race pure).

Il paraît judicieux de distinguer les pertes intervenant avant et après l'implantation car elles seraient distribuées indépendamment (ALLEN et al., J 947), ce qui va dans le sens de nos résultats où les génotypes se distinguent sur la seule mortalité pré-implantatoire (en émettant la réserve que notre étude de la mortalité postimplantatoire est incomplète). VENGE (I950) pense que pour un ovule normalement fécondé l'implantation dépend exclusivement des conditions physiologiques de la paroi utérine. ADAMs (I960, I962) attribue les pertes pré-implantatoires à un défaut de fécondation et à une sécrétion utérine inadéquate, alors que les pertes postimplantatoires résulteraient d'un développement placentaire imparfait ou d'une vascularisation déficiente. Il démontre d'ailleurs qu'en augmentant artificiellement. le nombre d'œufs fécondés, on augmente le nombre d'implantés, mais pas le nombre de nés vivants. Il suggère alors qu'une valeur plafond existerait chez une femelle, pour le nombre d'implantations qui peuvent être maintenues avec succès. Cette conclusion peut expliquer l'absence de différence entre les génotypes pour l'aptitude à avoir des embryons une fois le nombre de sites fixés.

Les effets liés au génotype de l'embryon ne paraissent pas induire des différences dans notre expérience (ceux-ci sont tous croisés double ou triple). Par contre, l'effet maternel paraît important du fait de l'effet d'hétérosis maternel de ro p. roo. Cela confirme les résultats de GrEGORy (I932) qui trouve un plus haut degré de viabilité ( $98 \mathrm{p}$. roo) chez les issus de femelles croisées que chez ceux de femelles de race pure ( $80 \mathrm{p}$. IOo). Cet effet peut s'expliquer par les meilleures capacités maternelles d'une femelle croisée, liées à un meilleur développement des organes maternels en cause lors du développement des jeunes jusqu'au sevrage.

Non significatifs, les effets "race de père " de la femelle (qui incluent des effets directs et maternels) apparaissent dans un classement contraire aux effets directs sur l'ovulation. Cela tendrait à montrer qu'il existe des interactions complexes entre les effets directs et maternels (peut être un antagonisme si l'on se réfère aux classements des effets des races). En conséquence, les effets génétiques à améliorer dans une sélection ayant pour objectif l'augmentation de la taille de portée pourrait dépendre de la race dont on dispose pour réaliser cette amélioration : il apparaît par exemple que la race Petite Russe a une faible taille de portée du fait d'un faible niveau ovulatoire. Par contre la race Californienne voit sa portée affaiblie par une mauvaise capacité maternelle utérine.

Des effets grands-maternels sur la viabilité embryonnaire semblent exister. Ils rendent compte de l'effet de l'environnement d'une future mère durant son. plus jeune âge, voire pendant sa vie utérine, sur son aptitude ultérieure à développer des portées. La race $N e ́ o-Z e ́ l a n d a i s e$ se distingue par rapport aux deux autres. L'origine de cet effet ne semble pas lié à des différences de tailles de portées dont sont issues les femelles mises en comparaison. En effet, nous avons élevé les femelles

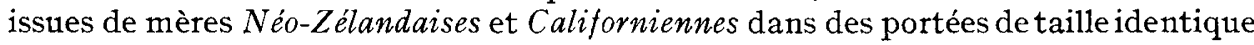
$(8,08$ et 8,24 respectivement $)$. Cela pourrait signifier que ces différences sont induites dès la vie utérine d'une jeune femelle. Nous aurions là l'exemple d'un 
effet très précoce dans la vie d'une femelle, conditionnant partiellement sa carrière ultérieure de reproductrice. Il existerait, en conséquence, une préparation optimale d'un futur reproducteur, combinant au mieux les effets utérins et postnataux jouant sans doute sur le développement général de l'animal considéré. Les liaisons entre ces effets et ceux précédemment indiqués n'apparaissent pas clairement ici. L'observation d'effets grands-maternels dans notre expérience peut être plus ou moins fortuite. En effet les animaux issus d'un type génétique (Néo-Zélandais $\times$ Petit Russe) sur les neuf mis en comparaison, ont la plus mauvaise viabilité (20 p. roo de pertes pré-implantatoires et un faible nombre d'ovules). Cet événement, pouvant être liés à des problèmes d'élevage lors de la fabrication des femelles de ce type génétique, entraîne deux conséquences :

- les effets grands-maternels n'apparaissent qu'à cause de ce génotype (viabilité identique pour tous les autres types génétiques croisés réciproques).

- du fait de la mauvaise performance enregistrée chez un des six types génétiques croisés, les effets d'hétérosis maternels sont en moyenne diminués.

\section{4. - Valeur synthétique de chaque race}

Le tableau 8 récapitule les effets génétiques considérés dans notre analyse pour les 3 races étudiées.

La race Californienne possède un niveau génétique direct élevé pour le nombre d'ovules pondus. Elle semble, de plus associer un effet génétique maternel favorable pour ce caractère, mais par suite d'effets " race de père " (combinant les effets directs et maternels) et d'effets grands-maternels défavorables, perd au cours du développement embryonnaire une grande partie des ovules pondus. La combinaison de ces effets conduit cette souche à avoir des tailles de portées de même ordre de grandeur que celle de la Néo-Zélandaise. Un effet d'hétérosis maternel de I 8 p. Ioo sur la viabilité pré-implantatoire tend à compenser les effets défavorables; cette race peut être considérée comme la meilleure race de père de femelle.

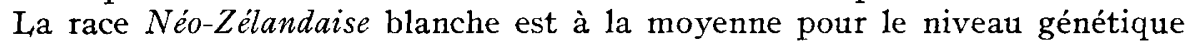
du nombre d'ovules pondus. Les effets génétiques directs et maternels sur la viabilité de ses ovules fécondés la mettent aussi en position intermédiaire. L'effet grand-maternel est le plus favorable après la saillie post partum. Cela conduit cette race à un équilibre entre des effets antagonistes qui lui permet d'être la meilleure race pure pour le critère synthétique représenté par la taille de portée. La prise en compte des effets de l'ascendance femelle (effet race de mère) la met en position favorable, ce qui nous permet de considérer cette race comme la meilleure race de mère de femelle.

Pénalisée par un faible niveau génétique d'ovulation (effets direct et maternel) la race Petite Russe se caractérise par son effet génétique global très favorable sur la viabilité préimplantatoire. Les effets grands-maternels défavorables ne lui permettent pas de maintenir son classement. Là encore, il semble que l'on soit arrivé à un équilibre naturel où une forte viabilité génétique compense la faible quantité d'ovules pondus. Cette race semble intéressante en croisement pour l'introduction par la voie mâle de ses gènes de viabilité et de son aptitude à l'ovulation.

Nous n'avons pas fait intervenir au niveau de la discussion le problème du degré de maturité de ces espèces, qui affecte le développement corporel et toutes les fonctions anatomiques et physiologiques. Certaines manifestations différentes entre souches au niveau de la fertilité ou de la prolificité ne sont-elles pas la conséquence de la plus grande maturité sexuelle présumée de la souche Petite Russe, 


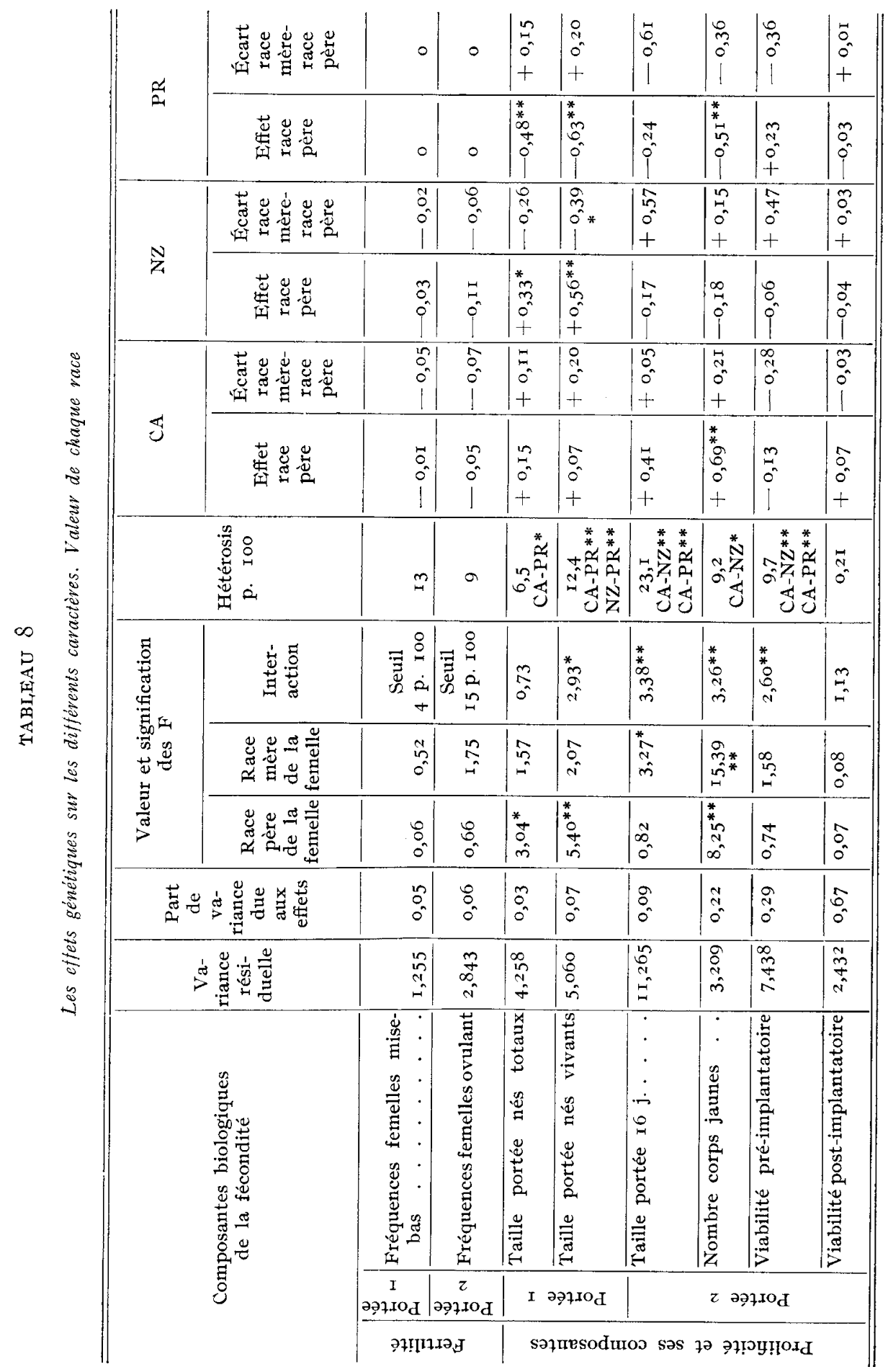


par exemple 1'aptitude à l'ovulation ou le meilleur maintien de la gestation. N'ayant aucune référence précise sur la " maturité sexuelle " des souches d'une part, nous situant pour l'étude des composantes à un âge moyen de 6 mois qui devrait atténuer les différences liées au jeune âge d'autre part, nous n'avons pas pu ici aborder cette question.

\section{5. - Incidence de la saillie post partum}

L'intensification du rythme de reproduction parait un moyen efficace et rapide pour augmenter la productivité numérique et dès I925 HAMMOND et MARSHALL, étudient l'aptitude de la lapine à ovuler et maintenir une gestation en saillie post partum dans des conditions particulières : nombre de petits réduit (lactation moins intense) ou alimentation améliorée, idée reprise et expérimentée par ADAms en I967. De nombreux travaux scientifiques ont suivi et tendent à conclure à une moins bonne fécondité de la lapine sans toutefois faire l'accord sur la composante responsable : subfertilité surtout liée à un défaut d'ovulation, SELME et PRUD'HON (I973), (68 p. Ioo de femelles ovulant en saillie post partum contre 84,6 p. Ioo chez le témoin), et Foxcroft et HASNAIN (I973) (o à 50 p. Ioo en saillie au jour o ou 3); subprolificité due à une ovulation déficiente (II,I corps jaunes en post partum et $\mathrm{r} 4 \mathrm{chez}$ le témoin) et à une fécondation anormale $(23 \mathrm{p}$. roo d'ovules non fécondés contre 5 p. IOO) selon TORRES et al. (I977), ou à une mortalité embryonnaire pré-implantatoire accentuée selon SELME et PRUD'HON (33 p. IOO en saillie post partum contre $22 \mathrm{p}$. roo chez le témoin), éventuellement post-implantatoire chez des femelles allaitantes selon HARNED et CASIDA, I969).

L'absence de témoin et l'aspect ponctuel de l'expérience (I portée) ne nous permettent pas de répondre à ces questions physiologiques ou de choisir le génotype le plus adapté à ce rythme de reproduction. Il apparaît toutefois à l'issue de la discussion de nos résultats que ceux-ci n'expriment pas de particularités réellement défavorables par rapport au rythme utilisé. Ce constat nous permet d'espérer que nos conclusions ne seront pas liées seulement à un modèle de reproduction mais peuvent exprimer une certaine généralité concernant les souches utilisées.

\section{Conclusion}

Nous avons dans cette expérimentation tenté d'estimer différents effets génétiques qui interviennent de façon conjointe sur des composantes du taux de gestation et de la taille de portée entre trois races de lapins, en les rapportant à la biologie de l'espèce.

En ce qui concerne la fertilité, qui est avant tout un problème d'aptitude à ovuler le plus souvent possible, on se heurte à un mécanisme complexa et encore mal connu : l'œstrus de comportement, sa correspondance avec le "cycle ovarien ", sa fréquence et les facteurs qui le conditionnent. La faculté de pondre souvent paraît " disjointe » de la faculté de pondre un grand nombre d'ovules (cas des souches de petit format) mais ces deux phénomènes ne sont peut-être pas nécessairement antagonistes chez un individu : cas de femelles dites " hyperfécondes " qui semblent répondre à des rythmes de reproduction intensif en alliant une taille de portée élevée (qui dépend implicitement d'un nombre moyen ou élevé d'ovules). Cette 


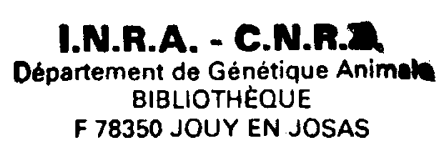

TAILLE DE LA PORTÉE DU LAPIN

diversité des mécanismes en cause pourrait expliquer la faible part des effets génétiques directs trouvés dans cette expérience en dépit de l'aptitude confirmée des génotypes de petit format et associés au petit format à une plus grande fertilité.

En ce qui concerne la prolificité, il nous semble hasardeux de vouloir interpréter la taille de portée I par les résultats de ses composantes en portée 2 et trouver une parfaite concordance dans l'interprétation des effets génétiques. La portée I est en effet caractérisée par une moins bonne expression des capacités de reproduction, une faible variabilité et paraît avoir peu de valeur prédictrice pour la future prolificité d'une femelle. Par ailleurs, à la lumière de nos résultats sur les composantes, il apparaît difficile de définir des effets génétiques précis sur un caractère dont la globalité masque en définitive les réelles possibilités de reproduction de chaque race (exemple du Petit Russe dont les qualités de bonne viabilité ne peuvent s'exprimer du fait du faible nombre d'ovules pondus). Enfin, l'analyse de la taille de portée fait abstraction de toutes les femelles dont 1'ovulation n'est pas suivie de parturition, réalité biologique non négligeable qu'il faut inclure dans une étude des effets génétiques afférant à la reproduction. Il conviendrait à ce sujet, pour être tout à fait rigoureux, de préciser chez les femelles ovulant l'origine de la non-gestation : mortalité embryonnaire précoce due aux gènes de l'embryon et (ou) à l'effet maternel de la femelle (comme nous l'avons interprété dans notre expérience) ou non-fécondation qui soulève à la fois le problème de l'effet maternel sur la qualité des ovules mais aussi celui du mâle quant à la fécondance de son sperme.

Il ressort de notre analyse que l'ovulation dépend d'un mécanisme génétique non uniquement additif et que deux de nos souches Californienne et $N$ éo-Zélandaise paraissent être caractérisées par des valeurs significativement différentes. Cet aspect important mérite confirmation et suggère des travaux plus analytiques au niveau de l'ovaire : une étude quantitative et qualitative des populations folliculaires est actuellement en cours. Celles-ci, à caractère peut-être plus héritable, sont d'une part en liaison avec la ponte ovulaire, source de prolificité potentielle, et d'autre part sous le contrôle direct des hormones de la reproduction (FSH) éventuels critères de sélection plus maniables.

Les effets moyens des gènes sont différents pour les caractères de viabilité où ils s'expriment sous forme d'effets maternels (additifs et d'hétérosis) et sur la seule viabilité pré-implantatoire. Nous ne pouvons pas ici séparer les effets additifs directs des effets additifs maternels. Il semble souhaitable, pour une meilleure interprétation de planifier une expérimentation les mettant en évidence. Cependant, l'étude partielle du nombre de fœetus à la mi-gestation ne permet pas de conclure sur une absence totale d'effet au cours de toute la vie embryonnaire post-implantatoire. L'ensemble des effets indirects rapportés dans cette expérience paraissent avoir une origine très précoce dans la vie d'une reproductrice (peut-être dès sa vie utérine). On peut alors penser que c'est par le biais de la précocité relative à chacun des types génétiques mis en comparaison, au moment où se sont faites les mesures, qu'apparaissent ces effets.

Les effets génétiques estimés sur les composantes du taux de gestation ou de la taille de portée, sont plus précis que sur le caractère global, cependant pour les utiliser à des fins de sélection il faut savoir à quel moment les mesurer dans la carrière d'une femelle. Dans le but d'étudier plus complètement cette question nous avons entrepris une étude, dans le temps, de ces composantes pour les deux souches Californienne et Néo-Zélandaise qui paraissent actuellement les plus intéressantes dans la pratique. Dès maintenant, par sélection il paraît possible d'espérer un progrès dans l'amélioration de certaines composantes en souche pure. 
La réalité non négligeable des effets maternels conduit à envisager au niveau des souches parentales une destinée paternelle ou maternelle en vue de la création d'une femelle croisée bénéficiant des bonnes performances parentales et d'un effet d'hétérosis de l'ordre de ro p. roo au niveau de chacune des composantes.

Au travers de ces résultats est démontrée l'efficacité des renseignements apportés par l'étude de caractères analytiques en vue d'acquérir une certaine maîtrise dans le choix de races, dans leur méthode de sélection et dans la stratégie de leurs croisements.

Reçu pour publication en mai I979.

\section{Remerciements}

Nous tenons à remercier tout particulièrement B. POUJARDIEU pour son aide précieuse notamment lors de l'initialisation de ce travail, ainsi que R. DUzer'T et J. SAPPA pour leur collaboration technique.

\section{Summary}

Analysis of genetic variations between three rabbit purebreeds for the litter size and
its biological components after a post partum mating

A laparotomy of 273 primiparous does from 9 genotypes, mated post partum by Fauves de Bourgogne bucks, gives us the values of the number of corpus luteus, implantation sites and embryos at 16 days of pregnancy. The 9 genotypes are 3 purebreeds (Californienne, NéoZélandaise blanche, Petite Russe) and the 6 reciprocal crosses obtained by pairing the 3 purebreeds. A genetical model shows the direct, maternal and gran maternal effects on the litter size and its components (numbers of corpora lutea (C. L.), loss of ova). The comparison between genetical effects taken in account for the gestation rate and litter size components indicates the superiority of the Californienne breed for the direct genetic effects on the corpora lutea, the ability of the Petite Russe breed for the direct genetic effects on the gestation and the viability of the embryos, the emphasis of the favourable effects for a Neo-Zélandaise doe due to her maternal ancestry. Heterotic direct effects of arround ro p. Ioo are pointed out for the corpora lutea. Maternal effects of heterosis from Io to $20 \mathrm{p}$. roo are found for the losses only before implantation. We try to discuss the genetical effects estimated by an anova in relation to the biological mecanisms involved.

\section{Références bibliographiques}

Adams C. E., I960a. Studies on prenatal mortality in the rabbit Oryctolagus Cuniculus : the amount and distribution of loss before and after implantation. J. Endocrin., 19, 325-344.

Adams C. E., I96ob. Prenatal mortality in the rabbit. J. Reprod. Fert., 1, 36.

ADAMS C. E., I962. Studies on prenatal mortality in the rabbit : effect of transfering vaying numbers of eggs. J. Endocrin., 24, 47 I-490.

ADAMS C. E., I965. The infuence of maternel environment on preimplantation stages of pregnancy, pp. 345-373. Ed. G.E.W.W. Wolstenholme and M. O'Connor. London : Churchill.

ADAMS C. E., I967. Concurrent Lactation and pregnancy in the rabbit. J. Reprod. Fert., 14, $35 \mathrm{I}-352$.

Alien P., Brambeli F. W. R., Milis I. H., I947. Studies on sterility and Prenatal mortality based on counts of corpora lutea, implantation sites and embryos. J. exp. Biol., 23, 312-317.

BRambel, F. W. R., I948. Prenatal mortality in mammals. Biol. Rev., 23, 370-407.

Foote R. H., Hafs R. E., Staples R. E., Gregorre A. T., BratTon R. W., I963. Ovulation rates and litter sizes in sexually receptive and non receptive artificially inseminated rabbits given varying dosages of luteinizing hormone. $J$. Reprod. Fert., 5, 59-66. 
Fox R. R., I968. Coitus as an ovulation stimulant in the laboratory rabbit. Proc. Soc. exp. Biol. Med., 128, 639-642.

FoxcRoft G. R., HASNAIN H., 1973. Time to mating after parturition on reproduction in the domestic rabbit $J$. Reprod. Fert., 33, 367-377.

GABRIEL K. R., I963. Analysis of variance of proportions with unequal frequencies $J$. Am. Statist. Ass., 58, I I 53-I 157 .

GrEgory P. W., I932. Potential and actual fecundity of some breeds of rabbits. J. Exp. Zool., 62, $27 \mathrm{I}-285$.

Hammond J., Marshal, F. H. A., 1925. Reproduction in the rablit, 2 I2 p. Oliver and Boyd. Edimburg.

Hammond J., I928. Die Krontrolle der fruchtbarkeit bei tieren - zuchungs kunde, 3, 523-547.

Hammond J., I933. The inheritance of fertility in the rabbit. Proc. 6th. Intern. Congr. Genet., 2, $7^{8-79}$.

HAmmond J., I934. Inheritances of fertility in the rabbit. Harper Adams Util. Poult. J., 19, $557-558$.

HARNED M. A., CASIDA L. E., 1969. Some post-partum reproductive phenomena in the domestic rabbit. J. Anim. Sci., 28, $785-788$.

HARVEY W. R., I975. Least Squares analysis of data with unequal subclass number. Agricultural Research Service. US. Department of Agriculture.

Hulot F., r975. L'insémination artificielle ne peut encore être généralisée. Revue de l'élevage $n^{0}$ hors-sévie, 5I-54.

Hulo't F., Poujardieu B., r 976 . Induction artificielle de l'ovulation et fertilité chez la lapine allaitante ou non. Ann. Biol. anim. Bioch. Biophys., 16, 635-643.

Matheron G., Poujardieu B., I976. Hétérosis pour quelques caractères de reproduction chez le lapin. Analyse de plans de croisement. Bull. Tech. Dép. Génét. Anim. Inst. natn. Rech. agron. Fr., no 24, 69-77.

PICKARD J. N., 1930. A preliminary study of some of the factors influencing the duration of pregnancy and litter size in the rabbit. Proc. 4 th. Worlds Poult. Congr., 901-909.

PoujARdieu B., VRILloN J. I., 1973. Variation de la productivité numérique au sevrage et de ses composantes entre génotypes de lapines croisées et de race pure. Jour. rech. avic. et cunic., décembre r973, 89-93. I.T.A.V.I.

Prud'hon M., Rouvier R., CAEI, J., BEL, I., I969. Influence de l'intervalle entre la parturition et la saillie sur la fertilité et la prolificité des lapins. Ann. Zootech., 18, 31 7-329.

RoBINSON R., I958. Genetics studies of the rabbit. Bibliographia Genetica XVIII, $229-554$.

Rosahn P. D., Grem H. S. N., Hu C. K., r934a. Hereditary variations in litter size. Proc. Soc. Exp. Biol. Med., 31, I2I4-I 2 I6.

Rouvier R., Poujardieu B., VRillon J. I., 1973. Analyse statistique des performances d'élevage des lapines. Facteurs de milieu, corrélations, répétabilités. Ann. Génét. Sél. anim., 5, (I), 83-107.

SAwIN P. B., I954. The influence of age of mother on pattern of productions. Ann. N. Y. Acad. Sci., 57, 564-574.

SElme M., PRUd'hon M., r 973. Comparaison au cours de différentes saisons des taux d'ovulation, d'implantation et de survie embryonnaire chez les lapines allaitantes saillies à l'œstrus postpartum et chez les lapines témoins. Jour. Rech. Avic. et Cunic., décembre 1973, 55-58, I.T.A.V.I.

Torres S., Gerard M., ThibaulT C., I977. Fertility factor in lactating rabbit mated 24 hours and 25 days after parturition. Ann. Biol. anim. Bioch. Biophys., 17 (1), 63-69.

VENGE O., x950. Studies of the maternal influence on the birth weight in rabbits. Acta Zoologica, 31, $1-48$. 